\title{
VOLUME DE FLUÍDO INTRAVENOSO E ALTA HOSPITALAR PRECOCE EM COLECISTECTOMIA ABERTA
}

\author{
VOLUME OF INTRAVENOUS FLUIDS AND EARLY HOSPITAL DISCHARGE AFTER OPEN \\ CHOLECYSTECTOMY
}

\author{
José Eduardo de Aguilar-Nascimento, TCBC-MT'; Alberto Bicudo-Salomão²; \\ Cervantes Caporossi, TCBC - MT $^{3}$; Raquel de Melo Silva ${ }^{4}$; \\ Eduardo Antonio Cardoso ${ }^{4}$; Tiago Pádua Santos ${ }^{4}$
}

\begin{abstract}
RESUMO: Objetivo: Investigar se a adoção de um protocolo de restrição hídrica intravenosa em colecistectomia aberta diminui a permanência hospitalar. Método: Estudo prospectivo envolvendo pacientes submetidas a colecistectomia aberta após a adoção de um protocolo multimodal (projeto ACERTO). Pacientes com intercorrencias intra- ou pós-operatórias foram excluídas. Todas as pacientes receberam uma solução de carboidratos $2 \mathrm{~h}$ antes da operação. Foram coletadas as seguintes variáveis: peso, altura, estado nutricional, volume total de fluidos intravenosos (VTFI) e volume prescrito em $\mathrm{ml} / \mathrm{kg}$ de peso por dia. Resultados: 64 pacientes $(11 \mathrm{M}$ e $53 \mathrm{~F}$; idade mediana $=43$ [18-65] anos) completaram o estudo. A re-alimentação pós-operatória ocorreu no mesmo dia da operação $(62,5 \%)$ ou no dia seguinte $(37,5 \%)$. Não foram registradas complicações infecciosas ou óbitos. O tempo mediano de internação pós-operatória foi de 1 (1-4) dia. Observou-se correlação entre o VTFI ( $\mathrm{r}=0,44 ; \mathrm{p}<0.001)$ e volume de fluídos / kg peso /dia $(\mathrm{r}=-0,29 ; \mathrm{p}=0,03)$ e o tempo de internação pós-operatória. Alta no $1^{\circ} \mathrm{PO}$ foi possível em $73,3 \%$ dos casos quando a prescrição foi de até $20 \mathrm{~mL} / \mathrm{Kg} / \mathrm{dia}$ e em $41,2 \%$ quando o volume prescrito foi maior ( $\mathrm{p}<0.001$; Odds Ratio=3,92; IC95\% 1,36-11,32). Conclusão: A restrição de fluidos intravenosos em colecistectomia aberta determina alta mais precoce (Rev. Col. Bras. Cir. 2007; 34(6): 381-384).
\end{abstract}

Descritores: Colecistectomia; Tempo de internação; Hidratação.

\section{INTRODUÇÃO}

Os pacientes submetidos à anestesia geral ou bloqueio têm uma veia puncionada e recebem fluidos intravenosos durante a operação. Após o término da operação, o cirurgião executa a prescrição do pós-operatório imediato e, nessa prescrição, é comum a continuidade do uso de fluidos intravenosos. O racional para essa rotina é a segurança de uma via intravenosa rápida e a hidratação do paciente. Diferentes regimes utilizados pelos anestesistas e cirurgiões asseguram uma infusão de cristalóides que tem relação com a duração da operação, o peso do paciente, sua condição clínica prévia e o seu tempo de jejum no pré-operatório ${ }^{1}$.

A colecistectomia é uma das operações mais comumente realizadas no mundo inteiro. Embora a videolaparoscopia seja a via de acesso mais apropriada, ainda é comum no Brasil a realização da colecistectomia por via aberta. Rego et al. ${ }^{2}$ por exemplo, em um hospital universitário de São Paulo, apresentam resultados de 243 colecistectomias realizadas em idosos. Nessa casuística, coletada entre 1992 e 1999, aproximadamente $50 \%$ dos pacientes foram operados pela via aberta. Na prescrição do pós-operatório imediato é comum a prática da hidratação com fluidos na faixa de 30-50 $\mathrm{ml} / \mathrm{kg}$ de peso por $\mathrm{dia}^{3}$. Isso determina a introdução de $2000 \mathrm{a}$ $3500 \mathrm{ml}$ de soro por dia nos pacientes e por conseguinte, uma grande sobrecarga de sódio. As vezes, mesmo em casos não complicados, essa prescrição continua pelo $1^{\circ}$ dia de pósoperatório.

Entretanto, o volume peri-operatório de fluidos intravenosos infundidos tem sido questionado nos últimos anos. Estudos clínicos têm mostrado que regimes de restrição de fluidos intravenosos associam-se a menor taxa de complicações pós-operatórias e encurtam a permanência hospita$1 a r^{4-5}$. A adoção de protocolos multimodais que envolvem a abreviação do jejum pré-operatório com solução de carboidratos duas horas antes da operação e re-alimentação precoce no pós-operatório também contribuem para a redução de fluidos intravenosos ${ }^{6-7}$. O projeto ACERTO implantado no nosso hospital universitário mostrou que a restrição de fluidos peri-operatórios é possível, segura e em conjunto com outras medidas multimodais diminui complicações e tempo de internação ${ }^{7}$. No entanto, não encontramos nenhum trabalho que enfocasse a adoção de regime de restrição de fluidos em colecistectomia. Com base na literatura corrente é possível especular que a diminuição de fluidos proporcione alta mais precoce em pacientes colecistectomizados. Dessa maneira, o

1. Professor Titular do Departamento de Cirurgia da FCM/UFMT.
2. Cirurgião do Serviço de Cirurgia Geral do Hospital Universitário Julio Muller da UFMT.
3. Professor Adjunto do Departamento de Cirurgia da FCM/UFMT.
4. Alunos de Medicina da FCM/UFMT. Bolsistas PIBIC/CNPq.
Recebido em 01/08/2007
Aceito para publicação em 02/10/2007
Conflito de interesses: nenhum
Fonte de financiamento: nenhuma
Trabalho realizado no Departamento de Clínica Cirúrgica da Faculdade de Ciências Médicas da Universidade Federal do Mato Grosso (UFMT). 
objetivo deste trabalho foi o de investigar se a adoção de um protocolo de restrição hídrica intravenosa em colecistectomia aberta diminui a permanência hospitalar.

\section{MÉTODOS}

O trabalho foi aprovado pelo Comitê de Ética em Pesquisa do HU Julio Muller da UFMT. Em um desenho prospectivo, planejou-se acompanhar todos os pacientes com diagnóstico de colecistopatia crônica calculosa, classificados como ASA (American Society of Anesthesiologists) I ou II e submetidos à colecistectomia eletiva, por via aberta, no Hospital Universitário Julio Muller entre julho de 2005 e setembro de 2006. Pacientes submetidos à exploração cirúrgica de vias biliares, com diagnóstico intra-operatório de colecistite aguda, ou submetidos, concomitantemente, a outro procedimento operatório foram excluídos. Com o intuito de observar apenas pacientes sem intercorrências, planejou-se também excluir pacientes nos quais fez-se necessário o uso de dreno intraabdominal.

Todos os pacientes foram submetidos à incisão subcostal (Kocher) e receberam antibioticoprofilaxia com cefalexina $1 \mathrm{~g}$ IV em dose única ou durante o pós-operatório imediato. O protocolo peri-operatório em todos os pacientes incluía jejum de 2 horas antes da operação com ingestão de $200 \mathrm{ml}$ de bebida contendo maltinodextrose a 12,5\% (Nidex $₫$; Nestlé, São Paulo, Brasil), antieméticos, analgésicos e realimentação precoce, se possível no mesmo dia da operação.

Foram coletadas as seguintes variáveis: peso, altura, estado nutricional pela avaliação subjetiva global, volume total de fluidos intravenosos (VTFI) e volume prescrito em $\mathrm{ml} /$ $\mathrm{kg}$ de peso. VTFI foi conceituado como o total de volume cristalóide recebido pelo paciente no pós-operatório. A variável de resultado principal foi o tempo de internação pós-operatório expresso em dias. Alta hospitalar foi autorizada quando o paciente estava recebendo alimentação oral sem vômitos e sentindo-se em condição de ir para seu domicílio. Os dados foram analisados estatisticamente pela correlação de Pearson e por curva de sobrevida de Kaplan-Maier. Estabeleceu-se em $5 \%(\mathrm{P}<0.05)$ o nível de significância estatística.

\section{RESULTADOS}

No período de estudo foram operados 84 pacientes. Não houve mortalidade. Destes, sete pacientes foram excluídos devido à exploração cirúrgica do colédoco e dez por uso de dreno de Penrose. Dos 64 pacientes restantes (11 M; 17,2\% e $53 \mathrm{~F}$; $82,8 \%$; idade mediana $=43$ [18-65] anos), oito (12,5\%) estavam desnutridos e $22(34.4 \%)$ eram obesos. A anestesia por bloqueio peri-dural $(31,48,4 \%)$ isolada ou combinada com geral $(22 ; 34,4 \%)$ foi o tipo de procedimento anestésico mais freqüentemente usado.

O tempo mediano de operação foi de 108 (45-220) minutos. A re-alimentação pós-operatória ocorreu no mesmo dia da operação $(n=40 ; 62,5 \%)$ ou no dia seguinte $(n=24 ; 37,5 \%)$. Não foram registradas complicações infecciosas. Não se utilizou sonda naso-gástrica no pós-operatório.
O tempo mediano de internação pós-operatória foi de 1 (1-4) dia. Não houve correlação entre o tempo de operação e o período de internação pós-operatória $(\mathrm{r}=0,21 ; \mathrm{p}=0,10)$. $\mathrm{O}$ volume prescrito para os pacientes variou de 2 a $65 \mathrm{ml} / \mathrm{kg}$ por dia com mediana de $19 \mathrm{~mL} / \mathrm{kg} / \mathrm{dia}$. O VTFI mediano foi de 2000 (500-8000 ml). Observou-se forte correlação entre o VTFI $(\mathrm{r}=0,44 ; \mathrm{p}<0.001)$ e volume de fluídos prescritos / $\mathrm{kg}$ de peso / dia ( $\mathrm{r}=-0,29 ; \mathrm{p}=0,03)$ e o tempo de internação pós-operatória. A Figura 1 mostra a probabilidade de alta até o $2^{\circ}$ dia de pósoperatório em relação ao volume total de fluidos intravenosos recebidos. Na Figura 2, apresenta-se a probabilidade de alta

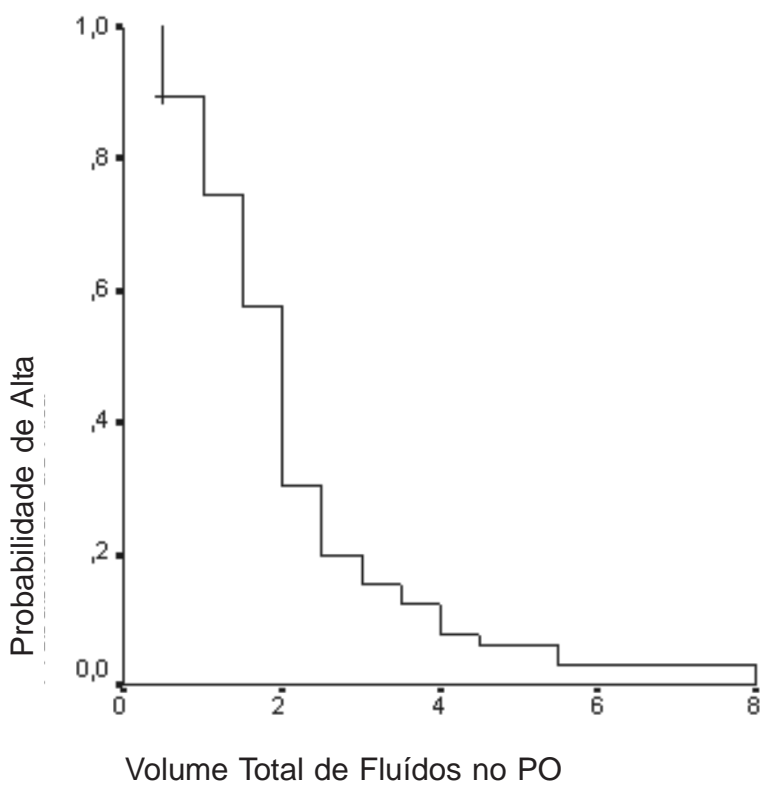

Figura 1 - Probabilidade de alta até o $2^{\circ}$ dia de pós-operatório em relação ao volume total de fluidos recebidos.

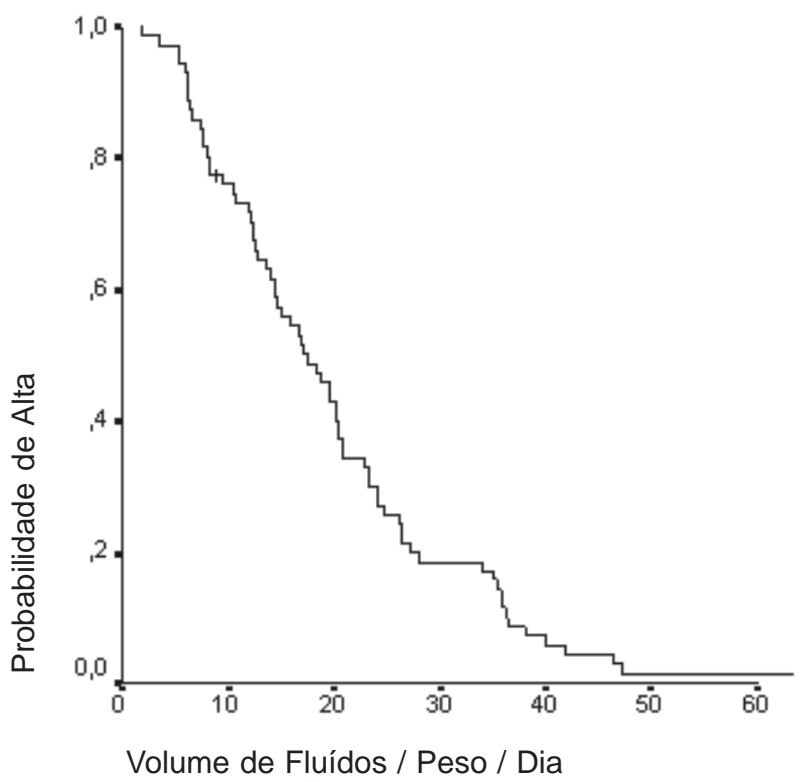

Figura 2 - Probabilidade de alta até o $2^{\circ}$ dia de pós-operatório em relação ao volume de fluídos recebidos $(m L)$ por quilo de peso por dia. 
em relação ao volume diário prescrito no pós-operatório. Observa-se nos dois gráficos que há nítida correlação entre o VTFI e o volume prescrito/kg de peso do paciente por dia com a probabilidade de alta precoce.

Alta no $1^{\circ}$ dia de pós-operatório foi possível em 73,3\% (22/30) dos casos quando a prescrição foi de até $20 \mathrm{ml} / \mathrm{Kg} / \mathrm{dia}$ e em 41,2\% (14/34) quando o volume prescrito foi maior ( $<<0.001$; Odds Ratio=3,92; IC95\% 1,36-11,32). Da mesma maneira, a probabilidade de alta mais precoce foi aproximadamente 3,5 vezes maior no Grupo que recebeu até $2000 \mathrm{~mL}$ no pós-operatório imediato (VTFI $<=2000 \mathrm{ml}=28 / 35 ; 80 \%$ vs. VTFI $=>2000 \mathrm{ml}=6 / 19 ; 31,6 \% ; \mathrm{p}=0.04 ;$ Odds ratio $=3,56$; IC95\% 1,14-11,15).

\section{DISCUSSÃO}

Os resultados deste trabalho mostram que a prescrição de fluidos intravenosos tem nítida relação com o tempo de internação em colecistectomia aberta. Num contingente de pacientes que evoluíram sem complicações infecciosas, o volume de soro infundido foi determinante para alta mais precoce. Pacientes que receberam em torno de $20 \mathrm{ml} / \mathrm{Kg} / \mathrm{dia}$ apresentaram chance de alta de aproximadamente quatro vezes maior que os que receberam mais fluidos intravenosos. Isso é relevante e aponta para a importância da restrição de fluidos nesses casos.

Na literatura atual, trabalhos randomizados e diretrizes (guidelines) de importantes sociedades apontam para a necessidade de restrição hídrica no peri-operatório ${ }^{5},{ }^{8}$. A sobrecarga de fluidos atrasa a recuperação dos pacientes pois tira a sede e a vontade de se alimentar9. Além disso, fluidos intravenosos podem diminuir a tensão de 0,2 nos músculos ${ }^{10} \mathrm{e}$ determinar ganho de peso, edema em alças intestinais aumentando o período de íleo adinâmico ${ }^{11}$. Uma prescrição convencional de um dia de pós-operatório em um paciente com laparotomia geralmente contem 2000 a $3000 \mathrm{ml}$ de fluido cristalino o que introduz no organismo três litros de água e de 154 a $308 \mathrm{mmol}$ de sódio por dia. Isso pode produzir uma sobrecarga de fluidos, aumento de peso e internação prolongada ${ }^{12}$.

Os dados apresentados no presente trabalho são importantes pois permitem uma análise com outro olhar sobre os resultados da implantação do projeto $\mathrm{ACERTO}^{7}$, antes desta implantação o Serviço já desenvolvia um protocolo de alta precoce após colecistectomia ${ }^{13}$. O projeto ACERTO, publicado em $2006^{7}$, introduziu mudanças no manuseio peri-operatório dos nossos pacientes tais como abreviação do jejum pré-operatório, restrição de fluidos intravenosos e realimentação precoce. No entanto, quando o protocolo de restrição de fluidos intravenosos não foi seguido, pacientes sem complicações evoluíram com necessidade de internação mais prolongada em virtude de mais náuseas, vômitos e sem vontade de ir para casa. Esses achados são particularmente importantes pois analisam um contingente de pacientes sem complicações pós-operatórias, sem ampliação da operação com abertura do colédoco e sem colocação de drenos, que são reconhecidamente razões para uma maior hospitalização. Tanto a curva de sobrevida em relação ao volume total de fluidos quanto ao volume prescrito baseado no peso do paciente mostraram uma relação significativa com a probabilidade de alta mais precoce. Pacientes com restrição de fluidos apresentaram-se mais adequados para receberem alta mais precoce. Com base nesses resultados, pode-se concluir que a restrição de fluidos em colecistectomia aberta determina alta mais precoce.

\begin{abstract}
Background: The aim of this stud y was to investigate the adoption of a protocol of intravenous fluid restriction in the length of hospital stay after open cholecystectomy. Method: Prospective study including female patients undergoing open cholecystectomy after the adoption of a new protocol (ACERTO protocol). Patients with intra- or postoperative interferences with original operation or normal course were excluded. All patients receive a carbohydrate beverage $2 h$ before operation. Variables collected were: weight, height, nutritional status, total volume of intravenous fluids (VTFI) and volume received in $\mathrm{ml} / \mathrm{kg}$ of weight/day. Results: 64 patients (11 M and $53 \mathrm{~F}$; median age $=43$ [18-65] years) entered the study. Postoperative re-feeding occurred in the same day of the operation (62.5\%) or in the next day (37.5\%). There were no deaths or postoperative infectious complications. Median length of postoperative stay was 1 (1-4) days. Discharge on the $1^{\text {st }} P O$ day was possible in $73.3 \%$ when prescription was at least $20 \mathrm{~mL} / \mathrm{kg} /$ day and in $41.2 \%$ when this volume was greater ( $(p<0.001$; Odds Ratio=3,92; IC95\% 1,36-11,32). Conclusion: Restriction of postoperative intravenous fluids leads to early hospital discharge after open cholecystectomy.
\end{abstract}

Key words: Cholecystectomy; Length of stay; Fluid therapy.

\section{REFERÊNCIAS}

1. Yamashita AM, Takaoka F, Auler JOC, Iwata NM. Anestesiologia. $5^{\text {a }}$ ed. São Paulo: Atheneu; 2001

2. Rêgo REC, Campos T, Moricz A, Silva RA, Pacheco Júnior AM. [Cholecystectomy in the elderly: early results of open versus laparoscopic approach]. AMB Rev Assoc Med Bras. 2003;49(3):293-9.
3. Andrade MAC, Martins P, Correia MITD, Rodrigues MAG. Hidratação venosa pós-operatória. In: Rodrigues MAG, Correia MITD, Rocha PRS, editores. Fundamentos em clínica cirúrgica. $1^{\mathrm{a}}$ ed. Belo Horizonte: Coopmed Editora Médica. 2005. p. 193-207.

4. Tambyraja AL, Sengupta F, MacGregor AB, Bartolo DC, Fearon KC. Patterns and clinical outcomes associated with routine intravenous sodium and fluid administration after colorectal 
resection. World J Surg. 2004;28(10): 1046-51; discussion 10512. Epub 2004 Sep 29.

5. Brandstrup B, Tønnesen H, Beier-Holgersen R, Hjorts $\varnothing$ E, Ørding $\mathrm{H}$, Lindorff-Larsen $\mathrm{K}$, et al. Effects of intravenous fluid restriction on postoperative complications: comparison of two perioperative fluid regimens: a randomized assessor-blinded multicenter trial. Ann Surg. 2003;238(5):641-8.

6. Brady M, Kinn S, Stuart P. Preoperative fasting for adults to prevent perioperative complications. Cochrane Database Syst Rev. 2003;(4):CD004423.

7. Aguilar-Nascimento JE, Bicudo-Salomão A, Caporossi C. Silva RM, Cardoso EA, Santos TP. Acerto pós-operatório: avaliação dos resultados da implantação de um protocolo multidisciplinar de cuidados peri-operatórios em cirurgia geral. Rev Col Bras Cir. 2006; 33(3):181-8.

8. Weimann A, Braga M, Harsanyi L, Laviano A, Ljungqvist O, Soeters P, DGEM (German Society for Nutritional Medicine), Jauch KW, Kemen M, Hiesmayr JM, Horbach T, Kuse ER, Vestweber KH; ESPEN (European Society for Parenteral and Enteral Nutrition). ESPEN Guidelines on Enteral Nutrition: Surgery including organ transplantation. Clin Nutr. 2006;25(2):224-44. Epub 2006 May 15.

9. Hausel J, Nygren J, Thorell A, Lagerkranser M, Ljungqvist O. Randomized clinical trial of the effects of oral preoperative carbohydrates on postoperative nausea and vomiting after laparoscopic cholecystectomy. Br J Surg. 2005;92(4):415-21.
10. Lang K, Boldt J, Suttner S, Haisch G. Colloids versus crystalloids and tissue oxygen tension in patients undergoing major abdominal surgery. Anesth Analg. 2001;93(2):405-9.

11. Lobo DN, Bostock KA, Neal KR, Perkins AC, Rowlands BJ, Allison SP. Effect of salt and water balance on recovery of gastrointestinal function after elective colonic resection: a randomised controlled trial. Lancet. 2002;359(9320):1812-8.

12. Lowell JA, Schifferdecker C, Driscoll DF, Benotti PN, Bistrian BR. Postoperative fluid overload: not a benign problem. Crit Care Med. 1990;18(7):728-33.

13. Aguillar-Nascimento JE, Caporossi C. Alta precoce em colecistectomia. Rev Col Bras Cir. 1992;19(3):152-5.

Como citar este artigo:

Aguilar-Nascimento JE, Bicudo - Salomão A, Caporossi C, Silva RM, Cardoso EA, Santos TP. Volume de fluído intravenoso e alta hospitalar precoce em colecistectomia aberta. Rev Col Bras Cir. [periódico na Internet] 2007; 34(6). Disponível em URL: http:// www.scielo.br/rcbc

Endereço para correspondência: José Eduardo de Aguilar Nascimento

Rua Estevão de Mendonça, 81 / 801

Goiabeiras

CEP: 78043-330 - Cuiabá - MT

E-mail: aguilar@terra.com.br 